\title{
Contaminación atmosférica, asma bronquial e infecciones respiratorias agudas en menores de edad, de La Habana
}

Manuel Romero-Placeres, M en C, (1) Pedro Más-Bermejo, MC, PhD, (1) Marina Lacasaña-N avarro, M en C, D ra en C,(2) Martha María Téllez Rojo-Solís, M en C, D ra en C, ${ }^{(2)}$ Juan A guilar-Valdés, MD, (1) Isabelle Romieu, MD, MPH, SCD. ${ }^{(2)}$

Romero-Placeres M, Más-Bermejo P, Lacasaña-Navarro M, Téllez Rojo-Solís MM,Aguilar-Valdés J, Romieu I. Contaminación atmosférica, asma bronquial e infecciones respiratorias agudas en menores de edad, de La Habana Salud Publica Mex 2004;46:222-233. El texto completo en inglés de este artículo está disponible en: http://www.insp.mx/salud/index.html

\section{Resumen}

Objetivo. Identificar la relación que guardan las consultas de urgencias por enfermedades respiratorias agudas, crisis agudas de asma bronquial e infecciones respiratorias agudas, con los cambios diarios en los niveles de contaminación atmosférica, en menores de 14 años de edad que fueron atendidos en dos centros hospitalarios de la ciudad de La Habana. Material y métodos Se realizó un estudio ecológico de series de tiempo durante el periodo comprendido entre el 1 de octubre de 1996 y el 11 marzo de 1998. Se estudió la asociación entre la presencia de crisis agudas de asma bronquial, infecciones respiratorias agudas y enfermedades respiratorias agudas por un lado, y la exposición a niveles de partículas menores de $10 \mu \mathrm{g} / \mathrm{m}^{3}\left(\mathrm{PM}_{10}\right)$, humo y dióxido de azufre ( $\mathrm{SO}_{2}$ ), por otro; asimismo, se construyeron modelos con la técnica de regresión binomial negativa, para estudiar periodos de latencia de 1 a 5 días y el efecto acumulado de siete días, previos a las consultas de urgencia. Resultados Los niveles de contaminación atmosférica, por lo general, resultaron bajos, ya que el promedio de 24 horas para PM , humo y SO fue de $59.2 \mu \mathrm{g} / \mathrm{m}^{3}$ ( $\left.D E=29.2\right), 27.7$ $\mu \mathrm{g} / \mathrm{m}^{3}\left(\mathrm{D}^{\prime} \mathrm{E}^{\prime}=21.2\right)$ y $21.1 \mu \mathrm{g} / \mathrm{m}^{3}(\mathrm{DE}=20.1)$, respectivamente. Un incremento de $20 \mu \mathrm{g} / \mathrm{m}^{3}$ en el promedio diario de humo se relacionó con un incremento de $2.2 \%$ (IC 95\% 0.9-3.6)

\author{
Romero-Placeres M, Más-Bermejo P, Lacasaña-Navarro M, \\ Téllez Rojo-Solís MM,Aguilar-Valdés J, Romieu I. \\ Air pollution, bronchial asthma, \\ and acute respirator and infections in children less years \\ of age, Habana City. \\ Salud Publica Mex 2004;46:222-233. \\ The English version of this paper \\ is available at: http://www.insp.mx/salud/index.html
}

\begin{abstract}
A bstract
Objective. To identify the relationship between emergency visits for acute respiratory illnesses, acute bronchial asthma crisis (ABAC) and acute respiratory infections (ARI), in children less than 14 years of age, and the daily changes of air pollutant levels, in two central hospitals of Havana City, between 0 ctober $1^{\text {st }} 1996$ and March 16 1998. Material and Methods. An ecological time series study was conducted.W e assessed the relationship between the presence of acute respiratory illnesses, $A B A C$, and IRA, and exposure to levels of particles less than $10 \mu \mathrm{g} / \mathrm{m}^{3}\left(\mathrm{PM}_{10}\right)$, smoke, and sulfur dioxide $\left(\mathrm{SO}_{2}\right)$; negative binomial regression models were used to assess latency periods of one to five days as well as the cumulative effect of seven days before the emergency visit. Results. The levels of atmospheric pollutants were low, in general.The 24-hour mean values for $\mathrm{PM}_{10}$ levels, smoke, and SO ${ }^{2}$, were $59.2 \mu \mathrm{g} / \mathrm{m}^{3}(\mathrm{SD}=29.2), 27.7 \mu \mathrm{g} / \mathrm{m}^{3}$ $(S D=21.2)$, and $21.1 \mu \mathrm{g} / \mathrm{m}^{3}(S D=20.1)$, respectively. $A n$ increase of $20 \mu \mathrm{g} / \mathrm{m}^{3}$ in the daily average of black smoke was associated with a $2.2 \%$ increase $(95 \% \mathrm{CI} \quad 0.9-3.6)$ in the number of emergency visits for ABAC.A $20 \mu \mathrm{g} / \mathrm{m}^{3}$ increase in the daily average of black smoke and $\mathrm{SO}_{2}$ was associated with an increase in ARI of $2.4 \%(95 \% \mathrm{Cl} 1.2-3.6)$, and $5 \%$ $(95 \% \mathrm{Cl} 1.3-5.3)$, respectively, with a 5 -day lag.W e also ob-
\end{abstract}

El protocolo fue subvencionado por la O rganización Panamericana de la Salud y el National Center for Enviromental Health de los Centers of Disease Control and Prevention, Atlanta, Georgia, Estados Unidos de América.

(1) Instituto $\mathrm{N}$ acional de Higiene, Epidemiología y Microbiología. La Habana, Cuba.

(2) Centro de Investigación en Salud Poblacional, Instituto N acional de Salud Pública. Cuernavaca, Morelos, México.

Fecha de recibido: 13 de mayo de 2003 - Fecha de aprobado: 20 de abril de 2004 Solicitud de sobretiros: D ra. Isabelle Romieu. Instituto N acional de Salud Pública. A venida Universidad 655 colonia Santa María A huacatitlán, 62508 Cuernavaca, Morelos, México. Correo electrónico: iromieu@ correo.insp.mx 
en el número de consultas de urgencias por crisis agudas de asma bronquial. Un incremento de $20 \mu \mathrm{g} / \mathrm{m}^{3}$ en el promedio diario de humo y de $\mathrm{SO}$, se relaciono con un incremento en las infecciones respirato rias agudas de $2.4 \%$ (95\% $\mathrm{Cl} 1.2-3.6)$ y $5 \%(95 \% \mathrm{Cl} 1.3-5.3)$, respectivamente, con un retraso de cinco días. Además, se presentó un efecto acumulado en todos los contaminantes estudiados. Conclusiones. Los resultados sugieren que los niveles de contaminantes atmosféricos en la ciudad de La H abana afec$\tan$ la salud respiratoria de los niños, por lo que se requiere de la aplicación de medidas de control, en particular para disminuir las emisiones vehiculares. El texto completo en inglés de este artículo está disponible en:http://www.insp.mx/ salud/index.html

Palabras clave: lesión por inhalación de humos; $\mathrm{SO}_{2}$; asma; infecciones del tracto respiratorio; niño; contaminación del aire; humo atmosférico; Cuba served a cumulative effect for all the contaminants studied. ConclusionsThese results suggest that air pollution levels in Havana City affect children's respiratory health; therefore implementation of control measures is necessary, especially those aimed at decreasing motor vehicle exhaust fumes.T he English version of this paper is available at: http:/ /www.insp.mx/salud/index.html

Key words: smoke inhalation injury; $\mathrm{SO}$; asthma; respiratory tract infections; child; air pollution; Cuba
$\mathrm{E}$ studios realizados en varios países han arrojado evidencias sobre la asociación entre los contaminantes atmosféricos y el incremento de las consultas de urgencias por crisis aguda de asma bronquial (CAAB) y otras enfermedades respiratorias. ${ }^{1,2}$ Diversos estudios epidemiológicos demuestran que la exposición a diferentes contaminantes, incluso a niveles situados por debajo de la norma, se asocian con un incremento en la incidencia y la severidad del asma, y con el deterioro de la función pulmonar, así como con otras enfermedades respiratorias en niños y adolescentes. ${ }^{3-8} \mathrm{La}$ relación entre la exposición a material particulado (humo y partículas menores a 10 micras) y los efectos adversos en la salud se ha documentado en muchos estudios, aunque sólo algunos han investigado el impacto del humo sobre la salud de niños con CAAB.

Los estudios realizados sobre este tema en Cuba, y en particular en la ciudad de La Habana, ${ }^{9-11}$ son escasos. Sin embargo, las estadísticas oficiales muestran prevalencias de asma bronquial elevadas; en 1998, por ejemplo, éstas eran de 91.1 por cada 1000 habitantes en la ciudad de La Habana. ${ }^{12}$ Específicamente, la prevalencia registrada para los municipios estudiados fue, en ese mismo año, de 141.4, 184.4, 196.6 por cada 1000 habitantes en los grupos de edad de 1 a 4,5 a 9 y 10 a 14 años en Centro Habana, respectivamente, y de 108.3, 143.8 y 176.7 para el municipio de Cerro. ${ }^{13}$ En la ciudad de La Habana existen grupos poblacionales expuestos a fuentes fijas de contaminantes atmosféricos que no cuentan con zonas de protección sanitaria (territorio ubicado en torno a las fuentes emisoras de contaminantes del aire, fuera de las zonas habitables por la población); se trata de industrias que se encuentran al norte y al sureste del área de estudio, y que cuentan con chimeneas de baja altura, lo que aumenta la acción contaminante (con material particulado) de sus actividades. Es preciso aclarar, además, que dichas industrias no cuentan con medidas de control para la disminución de la contaminación atmosférica. ${ }^{13}$ Por otra parte, los vehículos de automotor se encuentran en general en un estado técnico insatisfactorio por el largo tiempo que llevan en circulación (gran parte rebasa los 30 años) y por la falta de mantenimiento, lo cual genera un parque vehicular altamente contaminante, que aumenta potencialmente la contaminación del aire. ${ }^{11,13}$

Por resultados de estudios anteriores, al igual que por el incremento de las fuentes móviles y el elevado número de industrias existentes en la ciudad de La Habana, se consideró necesario investigar el papel que desempeña la contaminación atmosférica (humo, partículas de diámetro menor a 10 micras $-\mathrm{PM}_{10}-\mathrm{y}$ dióxido de azufre $-\mathrm{SO}_{2}$ - en el incremento del número de consultas de urgencias por enfermedades respiratorias agudas (ERA), específicamente por CAAB e infecciones respiratorias agudas (IRA).

\section{Material y métodos}

Con la aprobación de los Comités de Etica del Instituto Nacional de Higiene, Epidemiología y Microbiología, de Cuba, y del Instituto Nacional de Salud Pública de México, se efectuó un estudio ecológico de series de tiempo en el cual se revisaron las consultas de urgencias que el Sistema Nacional de Salud ofreció a menores de 14 años de edad, del 1 de octubre de 1996 al 13 de marzo de 1998; para ello, se consultaron los expedientes médicos del Hospital Pediátrico Centro 
Habana y del Hospital Pediátrico del Cerro, sitos en la ciudad de La Habana, Cuba.

La población de estudio quedó integrada por menores de edad que asistieron al servicio de urgencias de los dos hospitales y residían en Centro Habana y Cerro al momento del estudio. Ambos municipios son urbanos, con una carga de tráfico vehicular elevada y con diversas industrias distribuidas a lo largo de su territorio; su topografía es llana, con edificaciones altas, y constituyen la región más poblada de la ciudad (48 $500 \mathrm{hab} / \mathrm{km}^{2}$ o 170000 habitantes en $3.5 \mathrm{~km}^{2}$, de los cuales $18.1 \%$ son menores de 15 años de edad).

La información se obtuvo a partir de los registros de los pacientes que acudieron al servicio de urgencias de los dos hospitales mencionados y cuya residencia se encontraba a menos de $5 \mathrm{~km}$ del monitor de aire. Se obtuvo información sobre: fecha y tipo de consulta (primera vez o subsecuente), edad del paciente, domicilio particular, zona de residencia y diagnósticos de consulta, los cuales se clasificaron de acuerdo con la décima revisión de la Clasificación Estadística Internacional de Enfermedades y Problemas Relacionados con la salud (CIE-10). Los diagnósticos incluidos fueron IRA, CAAB y ERA. Dentro de las IRA, se consideraron las infecciones agudas de las vías respiratorias superiores (J00-J06), la influenza y las neumonías (J10-J18), otras infecciones agudas de las vías respiratorias inferiores (J20-J22) y bronquitis no especificada como aguda o crónica (J40). Para las CAAB se incluyeron el asma bronquial (J45) y el estado asmático (J46). El grupo de las ERA se conformó con la suma de las IRA y las CAAB. ${ }^{14}$

Las concentraciones diarias alcanzadas por los contaminantes estudiados $\left(\mathrm{PM}_{10}, \mathrm{SO}_{2}\right.$ y humo, este último llamado black smoke en otros estudios), producidos por las industrias y el tráfico vehicular, se obtuvieron de la estación de monitoreo ambiental del Instituto Nacional de Higiene, Epidemiología y Microbiología (INHEM); los valores diarios de dichas concentraciones se registraron en microgramos por metro cúbico $\left(\mu \mathrm{g} / \mathrm{m}^{3}\right)$. Los niveles de humo correlacionan muy bien con el contenido de carbón elemental en las partículas finas y se relacionan con la emisión proveniente de los vehículos diesel. ${ }^{15} \mathrm{El}$ control de calidad de las muestras se realizó utilizando las técnicas estandarizadas del Global Environmental Monitoring Program $(\mathrm{GEM}){ }^{16}$

Las variables meteorológicas, como la temperatura $\left({ }^{\circ} \mathrm{C}\right)$ y el porcentaje diario de humedad relativa (máxima, mínima y media), se obtuvieron de los archivos del Instituto de Meteorología de Cuba. Esta información se recopiló durante las mismas fechas que las correspondientes a las consultas por CAAB, IRA y ERA.
De esta manera, se conformó una base de datos con información ambiental, meteorológica y de salud, la cual permitió evaluar la asociación entre los niveles de contaminantes y las consultas en urgencias por los padecimientos mencionados.

El análisis estadístico de la información se realizó con el paquete Stata 5.0. Los valores faltantes de los contaminantes atmosféricos detectados se estimaron por regresión lineal $(6 \%)$, utilizando toda la información ambiental disponible de la serie de tiempo completa. Se generaron variables dicotómicas para los diferentes grupos de edad (menor de 1 año, de 1 a 4 años, de 5 a 9 años y de 10 a 14 años), así como indicadores de fin de semana, área de ubicación de los hospitales y meses más cálidos (abril a noviembre).

Después de realizar un análisis exploratorio de las variables de interés, se analizó la asociación existente entre las consultas de urgencia por ERA y los diagnósticos de CAAB e IRA, por un lado, y los cambios diarios de los niveles de contaminantes, por otro, utilizando un modelo de regresión Poisson. Este modelo se aplica a una variable dependiente de conteo que toma valores enteros positivos y pequeños en relación con el tamaño de la población de estudio, ya que, por lo general, sólo una parte de la población acude, en un día determinado, a demandar servicios. ${ }^{17-19}$

Con el fin de controlar las posibles tendencias y los patrones estacionarios, se construyeron modelos básicos para los tres padecimientos de estudio. En el caso de las ERA se utilizó un modelo conformado por variables indicadoras del área, el día y el año de estudio, el fin de semana, la estación del año y el grupo de edad; asimismo, se incluyó la media diaria de temperatura lineal y cuadrática, al igual que la humedad relativa máxima; también se aplicó un filtro de cuatro días utilizando el promedio móvil. Para las $\mathrm{CAAB}$, el modelo correspondiente se integró con variables indicadoras, tanto del área como del fin de semana, con las mediciones de temperatura media diaria y temperatura media diaria al cuadrado, así como con el grupo de edad, el año de estudio, el filtro de cuatro días y el total de población. El modelo correspondiente a las IRA incluyó: área, fin de semana, temperatura media diaria, temperatura media diaria al cuadrado, grupo de edad, días y años de estudio, y promedio móvil de cuatro días. Debido a que la variable dependiente mostró un comportamiento Poisson, con un parámetro de sobredispersión significativamente diferente de 1, se propuso como técnica alternativa la regresión binomial negativa, la cual es una generalización de la regresión Poisson que incorpora esta característica de sobredispersión. ${ }^{19}$ Se controló la presencia de autocorrelación en datos de consultas de urgencia filtrando los datos a 
través del promedio móvil de cuatro días usado como offset. ${ }^{17}$ La bondad de ajuste y la calidad de los modelos se probó por medio de técnicas diagnósticas estándar, utilizando los residuos de devianza.

\section{Resultados}

Durante el periodo de estudio se ofrecieron 44029 consultas a menores de 14 años de edad por CAAB, en los servicios de urgencias de los dos hospitales. El Hospital Pediátrico de Centro Habana atendió a 29805 pacientes (67.7\%), y el Hospital Pediátrico del Cerro, a 14 224 (32.3\%). En lo que se refiere a las IRA, se ofertó un total de 99441 consultas: en el Hospital Pediátrico de Centro Habana se atendió a 65489 pacientes (65.9\%), y en el Hospital Pediátrico del Cerro, a 33952 (34.1\%). Las estadísticas descriptivas de las visitas de emergencia por ERA se presentan en el cuadro I.

En las figuras 1 y 2 aparecen mapas de la zona de estudio, en los que se muestra la ubicación geográfica de los hospitales, del monitor de contaminantes del aire, así como de las fuentes fijas de contaminación y las vías principales de transporte.

En las figuras 3A y 3B se presentan, en un gráfico de series de tiempo, el número de consultas ofrecidas diariamente por CAAB, IRA y ERA, así como la serie de los tres contaminantes estudiados. Esta figura sugiere una posible asociación positiva entre los días de las consultas por estas enfermedades y los niveles que alcanzaron cada día las $\mathrm{PM}_{10}$, el $\mathrm{SO}_{2}$ y el humo.

El cuadro II resume las estadísticas descriptivas y los niveles de los contaminantes atmosféricos permisibles en Cuba, así como las variables meteorológicas. Durante el periodo de estudio, los niveles ambientales de humo y $\mathrm{SO}_{2}$ rebasaron, en la ciudad de La Habana, los valores máximos de contaminación del aire establecidos por la norma correspondiente $\left(40 \mu \mathrm{g} / \mathrm{m}^{3}\right.$ promedio de 24 horas) en 54 días (10.25\%) y 25 días $(4.74 \%)$, respectivamente. ${ }^{20} \mathrm{Al}$ comparar los niveles alcanzados por las $\mathrm{PM}_{10}$ con la norma Cubana, se puede observar que éstos se sobrepasan en 35 días (6.64\%). En este mismo cuadro se describen las características de las variables meteorológicas.

El humo y las $\mathrm{PM}_{10}$ se correlacionaron de manera significativa $(r=0.37, p>0.00)$. Lo anterior se debe a que el humo -resultado de los procesos de combustiónestá compuesto por partículas finas $<2.5 \mathrm{mg}$ que, en consecuencia, están incluidas en la medición de $\mathrm{PM}_{10}$ (partículas $<10 \mathrm{mg} / \mathrm{m}^{3}$ ). El SO $\mathrm{S}_{2}$ y la temperatura media $(r=0.17, p>0.00)$, así como esta última y la humedad máxima $(r=0.32, p>0.00)$ presentaron igualmente una correlación significativa. El $\mathrm{SO}_{2}$ y las partículas finas,

\section{Cuadro I \\ ESTADÍSTICAS DESCRIPTIVAS DE LAS VISITAS A URGENCIAS POR ENFERMEDADES RESPIRATORIAS AGUDAS. La Habana, Cuba, octubre de 1996 a marzo de 1998}

\begin{tabular}{lrrrrr} 
Visitas & $\begin{array}{c}\text { Total } \\
\text { de consultas }\end{array}$ & Media & $\begin{array}{c}\text { Desviación } \\
\text { estándar }\end{array}$ & Rango & Mediana \\
ERA & 143470 & 34.03 & 25.69 & $2-166$ & 27 \\
\hline CAAB & 44029 & 10.44 & 8.98 & $1-61$ & 8 \\
\hline IRA & 99441 & 23.60 & 18.70 & $1-121$ & 19
\end{tabular}

ERA: enfermedades respiratorias agudas

$C A A B$ : crisis aguda de asma bronquial

IRA: infecciones respiratorias agudas

Fuente: registros de datos de los departamentos de estadística del Hospital Pediátrico de Centro Habana y del Hospital Pediátrico del Cerro. La Habana, C uba

en cambio, no resultaron significativamente correlacionados.

En el cuadro III se presentan los riesgos relativos (RR) asociados a al número de días que tardan en hacerse manifiestos los efectos que producen los contaminantes sobre el número de consultas por ERA; fueron calculados por los rangos intercuartiles de frecuencias específicas de los contaminantes (humo, $\mathrm{SO}_{2}$ y $\mathrm{PM}_{10}$ ). El efecto más importante de las $\mathrm{PM}_{10}$ sobre las ERA se presentó después de cinco días. Se estima que, en el número de consultas por ERA, un incremento de $30 \mu \mathrm{g} / \mathrm{m}^{3}$ de $\mathrm{PM}_{10}$ produciría un aumento de $2.5 \%$ ( $R R=1.025$, IC95\% 1.018-1.030) después de cinco días. Asimismo, se calcula que un incremento de $20 \mu \mathrm{g} / \mathrm{m}^{3}$ de humo, ocasionaría un aumento de 3.1\% ( $R R=1.031$, IC95\% 1.026-1.037) en las consultas por ERA después de cinco días. El riesgo observado por la acción del humo fue mayor que el correspondiente a las $\mathrm{PM}_{10}$; aunado a ello, cuando se consideró el promedio de siete días, se observó que un incremento de $20 \mu \mathrm{g} / \mathrm{m}^{3}$ se asoció con un aumento de 4.2\% ( $R R=1.042$, IC95\% 1.031-1.053) en el número de consultas. Los niveles de $\mathrm{SO}_{2}$ se asociaron con un aumento en las visitas de urgencia hospitalaria por ERA de 3.0\% ( $R R=1.030$, IC95\% 1.021-1.038), cinco días después de que se presentara un incremento de $20 \mu \mathrm{g} / \mathrm{m}^{3}$ en el contaminante. El efecto registrado, considerando el promedio de siete días, fue de 2.1\% ( $R R=1.021$, IC95\% 1.008-1.034).

Cuando se estudió el grupo específico de las CAAB (cuadro IV) se observó que el incremento de $30 \mu \mathrm{g} / \mathrm{m}^{3}$ promedio diario en las $\mathrm{PM}_{10}$ tuvo un efecto significativo sobre las consultas por esos padecimientos cinco 


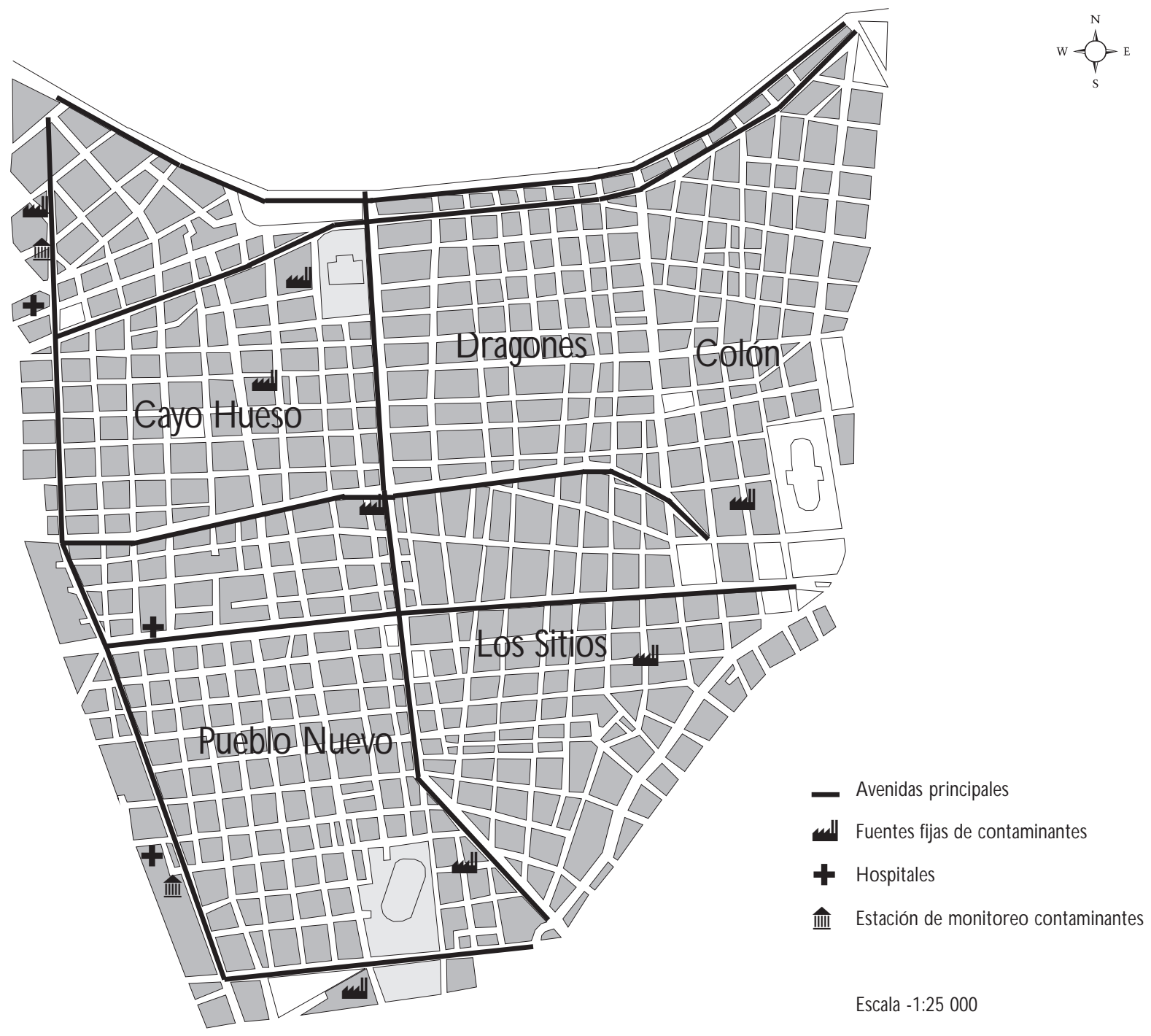

Figura 1. Fuentes contaminantes del aire. Municipio Centro Habana, Cuba, 1997

días después, pues se registró un aumento de $1.5 \%$ en las mismas (RR=1.015, IC95\% 1.001-1.029); no obstante el mayor efecto se observó cuando se consideró el promedio de siete días, ya que hubo un incremento -aunque no significativo- de $2.5 \%$, (RR=1.025, IC95\% 0.997-1.054). Asimismo, se observó que el número de visitas hospitalarias por $\mathrm{CAAB}$ tres días después de que el humo mostró un incremento de $20 \mu \mathrm{g} / \mathrm{m}^{3}$ promedio diario, aumentó 2.2\%, (RR=1.022 IC95\%: 1.0091.036). Los niveles de $\mathrm{SO}_{2}$ no mostraron relación alguna con las $\mathrm{CAAB}$ en el periodo de estudio.

En lo que corresponde a las IRA (cuadro V), un aumento de $20 \mu \mathrm{g} / \mathrm{m}^{3}$ en el promedio diario de humo, se asoció con un incremento de $2.4 \%$ en las consultas $(R R=1.024$, IC95\% 1.012-1.036\%) cinco días después.
Asimismo, se detectó una asociación significativa con un retraso en los efectos de cuatro días y con la exposición acumulada de siete días. La exposición a $\mathrm{SO}_{2}$ se asoció también con un incremento en el número de visitas de urgencia por IRA. Se observó un aumento de 3.3\% (RR=1.033, IC95\% 1.014-1.053) cinco días después de que los niveles del contaminante se elevaran, $y$ de $3.6 \%$ (RR=1.036, IC95\% 1.006-1.067) con el incremento de $20 \mu \mathrm{g} / \mathrm{m}^{3}$ en la exposición acumulada de siete días

\section{Discusión}

En este estudio, el incremento de los niveles de contaminantes atmosféricos, especialmente de humo, se 


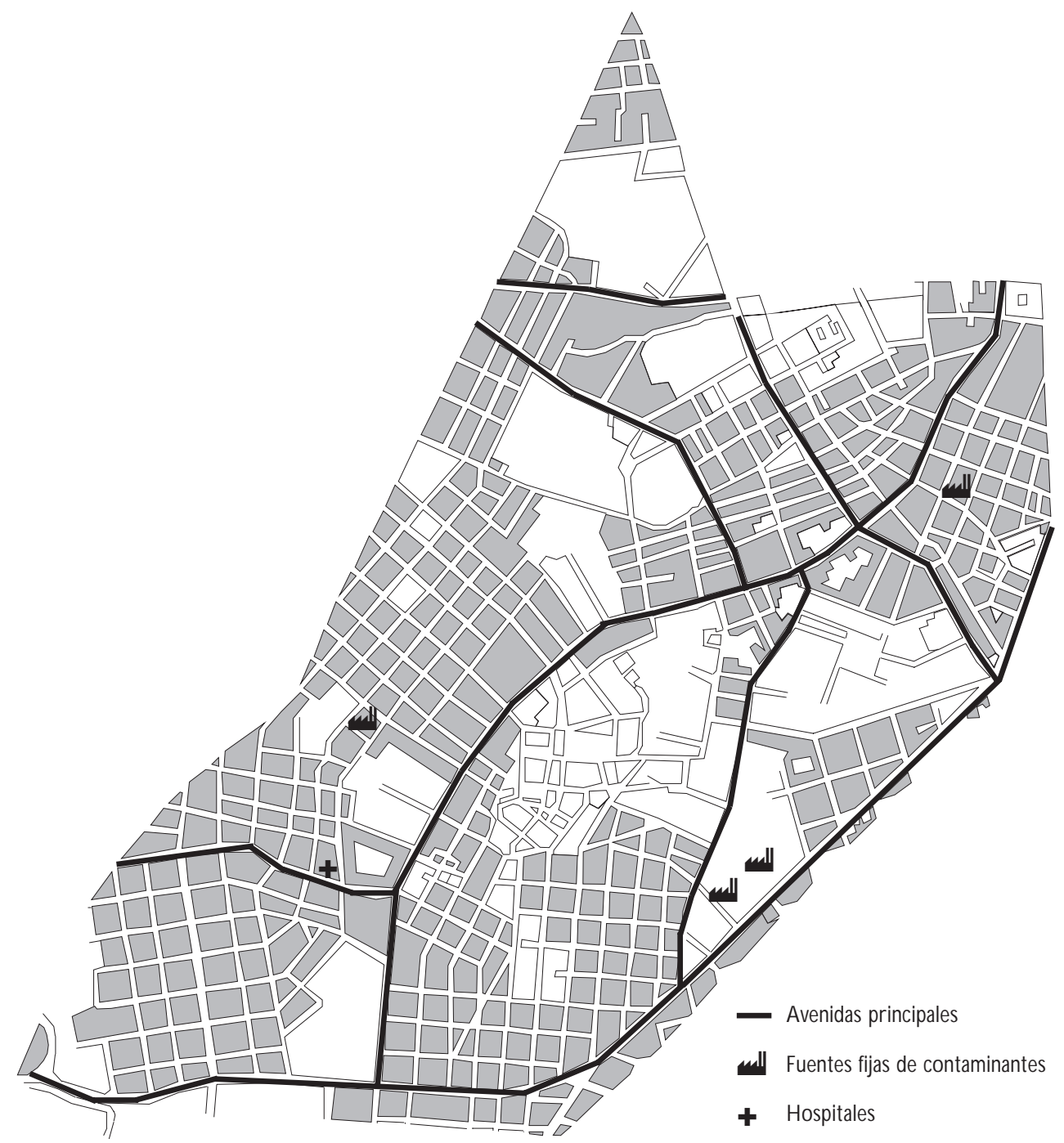

Figura 2. Fuentes contaminantes del aire. Municipio Cerro, Cuba, 1997

asoció con un aumento en las visitas de urgencia hospitalaria por ERA, CAAB e IRA en niños menores de 14 años de edad, después de controlar los efectos de las variables climatológicas y las tendencias seculares de las enfermedades. Los niveles de otros contaminantes del aire $\left(\mathrm{PM}_{10} \mathrm{y} \mathrm{SO}_{2}\right)$, también se asociaron con el número de visitas a urgencias; sin embargo, debido a la alta correlación entre humo y $\mathrm{PM}_{10}$, es difícil establecer los efectos en la salud de manera independiente. Esto explica el hecho de que la exposición a la mezcla de los contaminantes del aire, puede tener un efecto adverso en niños asmáticos e incrementar el riesgo de infecciones respiratorias.
En la ciudad de La Habana, el mayor problema de contaminación del aire se debe al material particulado, en especial al humo, que en el presente estudio excede la norma en $10.25 \%$ de los días. Esto se debe a que el combustible más utilizado para el transporte público en la ciudad es el diesel, el cual propicia un mayor escape de humo en los vehículos, que en su mayoría son antiguos. De acuerdo con datos de la Dirección de Operaciones de Cuba Petróleo, en la ciudad de La Habana el consumo de diesel y el de gasolina es de 100000 ton y 30000 ton por mes, respectivamente..$^{21}$ Estas cifras, además de ser elevadas, no representan el total del consumo de combustible, ya que existen otros distri- 

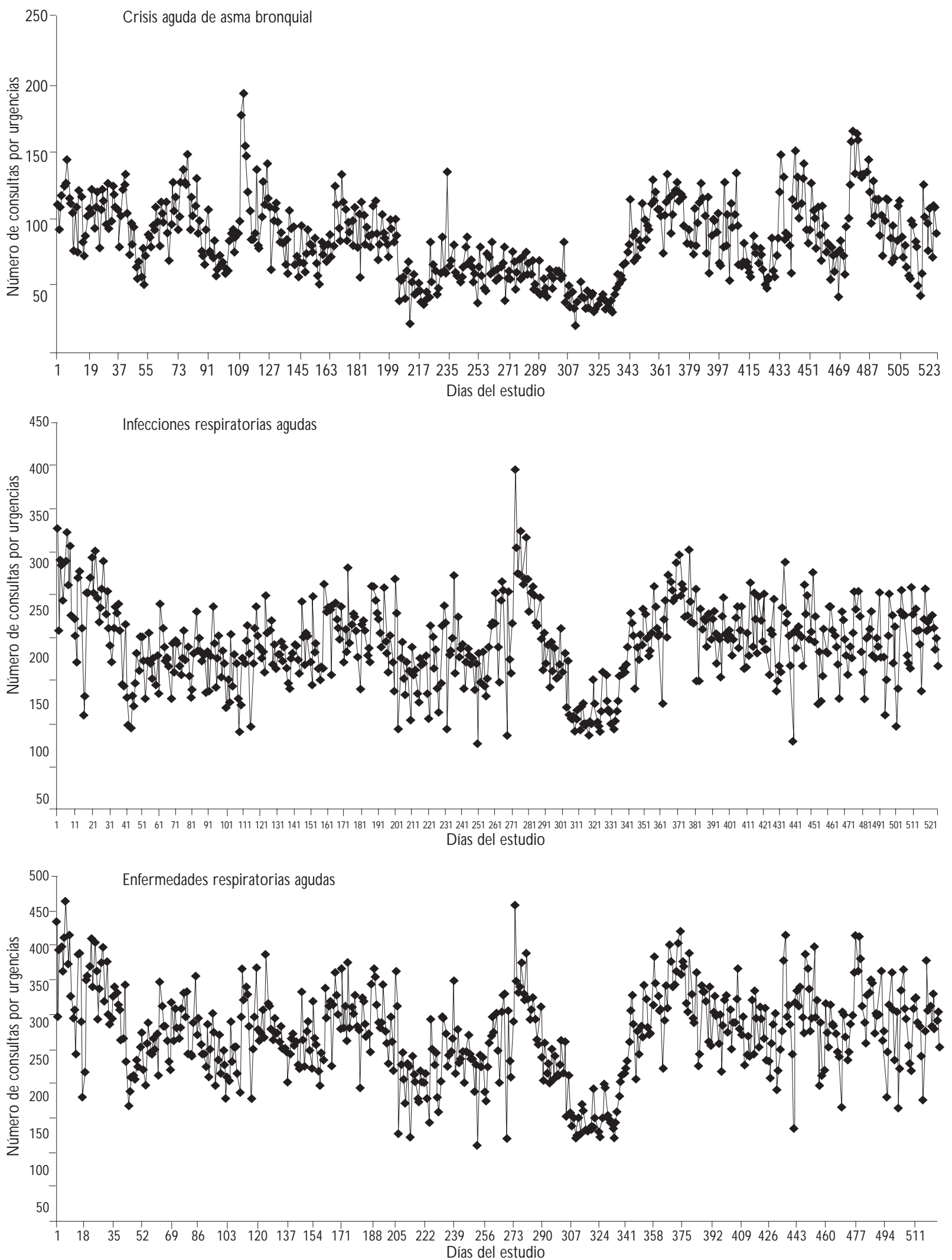

Figura 3A. Series de tiempo de las enfermedades respiratorias agudas. La Habana, Cuba, octubre de 1996 a MARZO DE 1998 

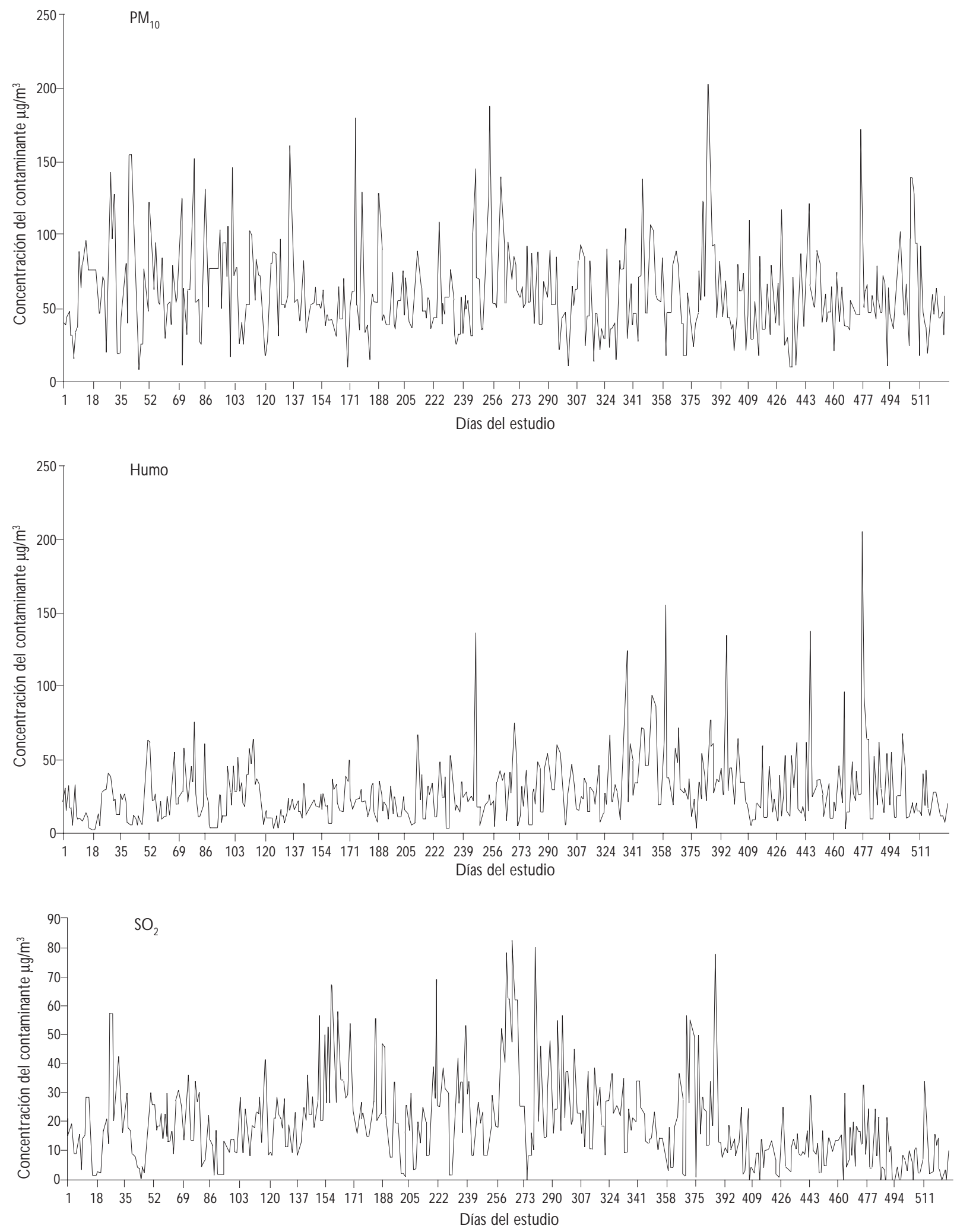

Figura 3B. Series de tiempo de los contaminantes estudiados. La Habana, Cuba, octubre de 1996 a marzo de 1998 


\section{Cuadro II \\ EstadíSTICAS DESCRIPTIVAS DE LOS CONTAMINANTES ATMOSFÉRICOS Y LAS VARIABLES METEOROLóGICAS en la zona de estudio. La Habana, Cuba, octubre de 1996 a marzo de 1998}

\begin{tabular}{|c|c|c|c|c|}
\hline Contaminantes atmosféricos y variables meteorológicas & Media & Desviación estándar & Rango & Mediana \\
\hline $\mathrm{PM}_{10}\left(\mu \mathrm{g} / \mathrm{m}^{3}\right)^{*}$ promedio $24 \mathrm{hrs}$. & 59.2 & 29.2 & $7.6-201.9$ & 53.8 \\
\hline Humo $\left(\mu \mathrm{g} / \mathrm{m}^{3}\right)^{*}$ promedio $24 \mathrm{hrs}$. & 27.7 & 21.2 & $2.4-204.2$ & 22.7 \\
\hline Dióxido de azufre $\left(\mu \mathrm{g} / \mathrm{m}^{3}\right)^{*}$ promedio $24 \mathrm{hrs}$. & 21.1 & 20.2 & $1.6-365.9$ & 18.0 \\
\hline Temperatura media $\left({ }^{\circ} \mathrm{C}\right) \ddagger$ promedio $24 \mathrm{hrs}$. & 24.8 & 2.48 & $15.4-29.4$ & 25.1 \\
\hline Humedad relativa máxima (\%)‡ promedio 24 hrs. & 90.9 & 6.36 & $63-100$ & 92.0 \\
\hline
\end{tabular}

* Registros de datos de la estación meteorológica de Casablanca, Instituto de Meteorología, Cuba

\section{Cuadro III \\ Coeficientes de Regresión para las enfermedades respiratorias agudas. La Habana, Cuba, OCTUBRE DE 1996 A MARZO DE 1998}

Variables Intercuartil RR Intervalo de confianza (95\%)

$\mathrm{PM}_{10}\left(\mu \mathrm{g} / \mathrm{m}^{3}\right)$

\begin{tabular}{cccc} 
Retrasos 1 & 30 & 1.000 & $0.994-1.005$ \\
\hline Retrasos 2 & 30 & 0.992 & $0.987-0.998$ \\
\hline Retrasos 3 & 30 & 0.985 & $0.979-0.990$ \\
\hline Retrasos 4 & 30 & 0.995 & $0.990-1.001$ \\
\hline Retrasos 5 & 30 & 1.025 & $1.018-1.030$ \\
\hline Promedio 7 & 30 & 1.018 & $1.007-1.030$
\end{tabular}

Humo $\left(\mu \mathrm{g} / \mathrm{m}^{3}\right)$

\begin{tabular}{cccc} 
Retrasos 1 & 20 & 1.009 & $1.004-1.014$ \\
\hline Retrasos 2 & 20 & 0.995 & $0.989-1.000$ \\
\hline Retrasos 3 & 20 & 0.996 & $0.991-1.001$ \\
\hline Retrasos 4 & 20 & 1.021 & $1.016-1.027$ \\
\hline Retrasos 5 & 20 & 1.031 & $1.026-1.037$ \\
\hline Promedio 7 & 20 & 1.042 & $1.031-1.053$
\end{tabular}

$\mathrm{SO}_{2}\left(\mu \mathrm{g} / \mathrm{m}^{3}\right)$

\begin{tabular}{llll} 
Retrasos 1 & 20 & 1.009 & $1.001-1.018$ \\
\hline Retrasos 2 & 20 & 0.991 & $0.983-1.000$ \\
\hline Retrasos 3 & 20 & 0.995 & $0.986-1.004$ \\
\hline Retrasos 4 & 20 & 1.004 & $0.997-1.013$ \\
\hline Retrasos 5 & 20 & 1.030 & $1.021-1.038$ \\
\hline Promedio 7 & 20 & 1.021 & $1.008-1.034$
\end{tabular}

Nota: ajustado por área, fin de semana/entre semana, grupos de edad, temperatura media diaria, temperatura media diaria al cuadrado, humedad máxima diaria, estación, días y años de estudio, promedio móvil de cuatro días

RR: riesgo relativo calculado para un incremento igual al rango intercuartílico (intercuartil)

$R R=\exp \{(r . i) * b e t a\}$

Retrasos: es la medición promedio del contaminante $(X)$ diario correspondiente a los $(Y)$ días anteriores al evento respiratorio
Cuadro IV

Coeficientes de Regresión PARA LAS CRISIS AGUdAS de asma bronquial. La Habana, Cuba, OCTUBRE DE 1996 A MARZO DE 1998

Variables

Intercuartil $\quad$ RR Intervalo de confianza (95\%)

$\mathrm{PM}_{10}\left(\mu \mathrm{g} / \mathrm{m}^{3}\right)$

\begin{tabular}{llll} 
Retrasos 1 & 30 & 1.000 & $0.987-1.014$ \\
\hline Retrasos 2 & 30 & 1.006 & $0.993-1.021$ \\
\hline Retrasos 3 & 30 & 1.007 & $0.993-1.021$ \\
\hline Retrasos 4 & 30 & 1.011 & $0.997-1.025$ \\
\hline Retrasos 5 & 30 & 1.015 & $1.001-1.029$ \\
\hline Promedio 7 & 30 & 1.025 & $0.997-1.054$
\end{tabular}

Humo $\left(\mu \mathrm{g} / \mathrm{m}^{3}\right)$

\begin{tabular}{llll} 
Retrasos 1 & 20 & 1.010 & $0.997-1.022$ \\
\hline Retrasos 2 & 20 & 1.015 & $1.002-1.028$ \\
\hline Retrasos 3 & 20 & 1.022 & $1.009-1.036$ \\
\hline Retrasos 4 & 20 & 1.006 & $0.993-1.019$ \\
\hline Retrasos 5 & 20 & 1.018 & $1.005-1.031$ \\
\hline Promedio 7 & 20 & 1.054 & $1.028-1.080$
\end{tabular}

$\mathrm{SO}_{2}\left(\mu \mathrm{g} / \mathrm{m}^{3}\right)$

\begin{tabular}{cccc} 
Retrasos 1 & 20 & 0.987 & $0.966-1.008$ \\
\hline Retrasos 2 & 20 & 0.985 & $0.964-1.006$ \\
\hline Retrasos 3 & 20 & 0.977 & $0.956-0.998$ \\
\hline Retrasos 4 & 20 & 0.984 & $0.963-1.006$ \\
\hline Retrasos 5 & 20 & 0.999 & $0.978-1.020$ \\
\hline Promedio 7 & 20 & 0.977 & $0.946-1.008$
\end{tabular}

N ota: ajustado por área, fin de semana/entre semana, temperatura media diaria, temperatura media diaria al cuadrado, grupos de edades, años del estudio, promedio móvil de cuatro días, total de población

RR: riesgo relativo calculado para un incremento igual al rango intercuartílico (intercuartil)

$\mathrm{RR}=\exp \{(\mathrm{r} . \mathrm{i}) *$ beta $\}$ 


\section{Cuadro V \\ Coeficientes de regresión para las infecciones respiratorias agudas. La Habana, Cuba, OCTUBRE DE 1996 A MARZO DE 1998}

\begin{tabular}{lccc} 
Variables & Intercuartil & RR & Intervalo de confianza (95\%) \\
& & & \\
$\mathrm{PM}_{10}\left(\mu \mathrm{g} / \mathrm{m}^{3}\right)$ & & & \\
Retrasos 1 & 30 & 0.995 & $0.983-1.008$ \\
\hline Retrasos 2 & 30 & 0.988 & $0.976-1.001$ \\
\hline Retrasos 3 & 30 & 0.980 & $0.967-0.992$ \\
\hline Retrasos 4 & 30 & 0.985 & $0.973-0.997$ \\
\hline Retrasos 5 & 30 & 1.013 & $1.001-1.026$ \\
\hline Promedio 7 & 30 & 0.995 & $0.971-1.020$ \\
& & & \\
Humo $\left(\mu \mathrm{g} / \mathrm{m}^{3}\right)$ & & & \\
Retrasos 1 & 20 & 1.007 & $0.995-1.019$ \\
\hline Retrasos 2 & 20 & 0.993 & $0.982-1.005$ \\
\hline Retrasos 3 & 20 & 0.991 & $0.979-1.003$ \\
\hline Retrasos 4 & 20 & 1.020 & $1.008-1.033$ \\
\hline Retrasos 5 & 20 & 1.024 & $1.012-1.036$ \\
\hline Promedio 7 & 20 & 1.025 & $1.002-1.048$ \\
\hline SO ( $\mu$ gg/m $\left.{ }^{3}\right)$ & & & \\
Retrasos 1 & 20 & 1.014 & $0.995-1.034$ \\
\hline Retrasos 2 & 20 & 0.999 & $0.980-1.019$ \\
\hline Retrasos 3 & 20 & 1.005 & $0.986-1.025$ \\
\hline Retrasos 4 & 20 & 1.011 & $0.992-1.031$ \\
\hline Retrasos 5 & 20 & 1.033 & $1.014-1.053$ \\
\hline Promedio 7 & 20 & 1.036 & $1.006-1.067$
\end{tabular}

N ota: ajustado por área, fin de semana/entre semana, temperatura media diaria, temperatura media diaria al cuadrado, grupos de edades, años del estudio, tiempo del estudio, promedio móvil de cuatro días

RR: riesgo relativo calculado para un incremento igual al rango intercuartílico (intercuartil)

$\mathrm{RR}=\exp \{(\mathrm{r} . \mathrm{i}) *$ beta $\}$

buidores de gasolina y diesel en la ciudad. Una de las fuentes más importantes de contaminación encontradas en la zona de estudio es el flujo vehicular.

Una investigación realizada durante 1996 en siete avenidas del área de estudio, notificó una media de 145 vehículos por hora, cifra considerada en esa fecha como moderada. Lo anterior se ve reflejado en los bajos valores de contaminantes que la red de monitoreo registró en ese año y hace suponer que, con el incremento de vehículos que ha habido en la ciudad últimamente, dichos valores se han elevado. ${ }^{13}$ Otras posibles fuentes de contaminación son el humo y las partículas derivadas de los procesos industriales, lo que puede estar relacionado con la ineficiencia en los procesos de combustión, o bien, con la ausencia o ineficiencia del control de las emisiones al ambiente de dichos contaminantes del aire. ${ }^{13,22}$

Los resultados del presente estudio muestran que el humo, el $\mathrm{SO}_{2}$ y las $\mathrm{PM}_{10}$ se asocian con el número de consultas por ERA, CAAB e IRA; ello coincide con otros estudios, los cuales refieren un efecto adverso de los contaminantes atmosféricos sobre el sistema respiratorio del individuo. Además, las concentraciones de $\mathrm{PM}_{10}$ y humo mostraron una asociación con la incidencia de ERA en niños escolares, lo que se hizo más evidente en aquellos menores con diagnóstico de asma bronquial. ${ }^{1,3,23}$

Resultados similares se observan en otros estudios sobre enfermedades respiratorias y contaminación atmosférica. ${ }^{24-31}$ En una investigación realizada en Francia, se encontró que existía una asociación entre estos padecimientos y niveles bajos de concentración de partículas y humo. ${ }^{32}$ Otro estudio hecho en Londres, encontró una asociación del humo con el retraso de un día en los efectos y el acumulado de tres días. ${ }^{33}$ Sin embargo, pocos estudios han evaluado el impacto de los contaminantes sobre las infecciones respiratorias agudas, ${ }^{3,34}$ y nuestros resultados sugieren que los niveles permitidos en las normas internacionales no protegen a los niños del efecto adverso que producen los contaminantes del aire sobre las funciones respiratorias.

Por otro lado, se deben considerar algunas limitaciones inherentes al diseño ecológico; no se contó con información individual que permitiera hacer una caracterización precisa del nivel de exposición, por lo que la exposición intradomicilaria a contaminantes y otros factores no fueron considerados, asumiendo que los niños tienen conductas similares y homogéneas. Esto pudo haber producido un error de clasificación no diferencial en la exposición y, en consecuencia, una subestimación del efecto.

Una de las ventajas del presente estudio fue que permitió obtener el número de visitas de urgencia que se atendieron por enfermedades respiratorias agudas (143 470) -lo que fue muy importante- y estandarizar los criterios diagnósticos con base en exámenes médicos completos, realizados por especialistas, lo que redujo la posibilidad de una mala clasificación de los padecimientos. Adicionalmente, ni la población que solicita el servicio ni los médicos de asistencia tienen conocimiento sobre los niveles de contaminación, por lo que el sesgo de información que pudiera existir es de tipo no diferencial. Asimismo, se revisó la calidad de los datos capturados en el momento de las visitas de urgencia, cotejándolos con los registros médicos diarios; así, se encontró menos de $2 \%$ de error en los registros. 
Los resultados sugieren que aun los bajos niveles de contaminación del aire en las ciudades son dañinos para el tracto respiratorio de los menores de edad y que ello se relaciona con el aumento del riesgo de padecer ERA, en particular CAAB. Es probable que una gran parte de los efectos adversos observados se relacionen con la exposición a humo. Estos problemas de contaminación del aire producen un gran impacto en la salud pública por la demanda de servicio que generan. Se calcula que una disminución de $20 \mu \mathrm{g} / \mathrm{m}^{3}$ en las concentraciones de humo y de $30 \mu \mathrm{g} / \mathrm{m}^{3}$ en las concentraciones de $\mathrm{PM}_{10}$, repercutiría en una disminución de la demanda de $2.2 \%$ en las consultas de urgencia por asma y de $3.6 \%$ en las correspondientes a IRA. Por último, es importante resaltar que los efectos adversos sobre la salud respiratoria de los niños se observaron incluso cuando los niveles de contaminantes estaban situados por debajo de los valores máximos establecidos por las normas cubanas vigentes.

\section{Referencias}

1. D ockery DW, Pope III CA.Acute respiratory effects of particulate air pollution. Environmental Epidemiology Program, Harvard School of Public Health, Boston, Massachusetts. Annu Rev Public Health 1994;15:107-132.

2. Bello S, 0 yarzún M. Efectos adversos para la salud de los contaminantes atmosféricos. Rev Chil Enferm Respir 1991;7:198-205. 3. Ilabaca M, 0 laeta I, Campos E,Villaire J,Téllez-Rojo MM, Romieu I. Association between levels of fine particulate and emergency visits for pneumonia and other respiratory illnesses among children in Santiago, Chile. J Air W aste Manage Assoc 1999;49:174-185.

4. Romieu I, Meneses F, Ruiz S, Sienra JJ, Huerta J,W hite MC et al. Effect of air pollution on the respiratory health of asthmatic children living in Mexico City. Am J Respir Crit Care Med 1996;154:300-307.

5. D ockery D, Speizer F, Stram D,W are J, Spengler J, Ferris B. Effects of inhalable particles on respiratory healths of children. Am Rev Respir D is 1989;139:587-594

6. Sánchez J, Romieu I, Ruiz S, Pino P, Gutiérrez M. Efectos agudos de las partículas respirables y del dióxido de azufre sobre la salud respiratoria en niños del área industrial de Puchuncavi, Chile.Am J Public Health 1999;6:384-391.

7. Hernández-C adena L,Téllez-Rojo MM, Sanín-A guirre LH, Lacasaña$\mathrm{N}$ avarro M, Campos A, Romieu I. Relationship between emergency consultations for respiratory disases and air pollution in Juarez City, Chihuahua. Salud Publica Mex 2000;42(4):288-297.

8. Barnes PJ.Air pollution and asthma. Postgrad Med J 1994;823: 319-325.

9. Del Puerto C, Molina E. La contaminación del aire y sus riesgos para la salud. En: Instituto $\mathrm{N}$ acional de Higiene, Epidemiología y

Microbiología, ed. Contaminación del aire y salud. México, DF: Ciencias Médicas; 1992:1-73 (Serie Salud Ambiental, N 0. 2).

10. Molina E, Bonito LA, CabreraA. Prevalencia de asma bronquial. A sociación con la contaminación atmosférica y otros efectos ambientales. En: Instituto $\mathrm{N}$ acional de Higiene, Epidemiología y

Microbiología, ed. Contaminación del aire y salud. México, DF: Ciencias Médicas; 1992:93-108 (Serie Salud A mbiental, N 0. 2).

11. González P. Contaminación atmosférica secundaria en una zona de Centro Habana (tesis). La Habana: Facultad de Biología-Universidad de La Habana; 1989.

12. Ministerio de Salud Pública de Cuba.Anuario estadístico 1998. La Habana: Artes Gráficas; 1998:72-73.

13. Molina E. Contaminación atmosférica en Centro Habana. A sociación con la morbilidad por asma bronquial y enfermedades respiratorias agudas (tesis). La Habana: Instituto Superior de Ciencias Médicas de La Habana; 1998.

14. O rganización Mundial de la Salud. C lasificación Estadística Internacional de Enfermedades y Problemas Relacionados con la Salud. Décima revisión.W ashington, D C: O PS; 1995:491-521. (Publicación Científica, N 0.554).

15. Hoek G, Forsberg F, Borowska M, H lawiczka S, Vaskövi E,W elinder H et al.W intertime $\mathrm{PM}_{10}$ and black smoke concentrations across Europe: Results from the peace study.Atmospheric Environment 1997;31(21):3609-3622.

16. W orld Health 0 rganization. A nalyzing and interpreting air monitoring data. GEMS. Ginebra:W HO ; 1980.

17. Schwartz J, Spix C, Toulomi G, Bachárova L, Barumamdzadeh T, Le Tertre A et al. Methodological issues in studies or air pollution and daily count of deaths or hospital admissions.J Epidemiol Community Health 1996;50(Suppl 1):S3-S11.

18.Téllez-Rojo MM, Romieu I, Ruiz-Velazco S, Meneses-González F, Hernández-A vila M. Efecto de la contaminación ambiental sobre las consultas por infecciones respiratorias en niños de la Ciudad de México. Salud Publica Mex 1997;39:513-522.

19. Lawless JF. N egative binomial and mixed poisson regression. C an J Stat 1987;15:209-225.

20. Comité Estatal de N ormalización. N ormas para la protección del medio ambiente.Atmósfera. Requisitos higiénico sanitarios: concentraciones máximas admisibles, alturas mínimas de expulsión y zonas de protección sanitaria. La Habana: CEN ; 1987 (N C:93-02-202). 21. Cuba Petróleo. Informe de la Dirección de 0 peraciones. La Habana: CUPET: 1999.

22. Gutiérrez JH, Romieu I, Corey G, Fortoul T. Contaminación del aire, riesgos para la salud. México, DF: UN AM/El Manual Moderno; 1997:4-5. 23. Hernández-C adena L,Téllez-Rojo MM, Sanín-A guirre LH, Lacasaña$\mathrm{N}$ avarro M, Campos A, Romieu I. Relación entre consultas a urgencias por enfermedad respiratoria y contaminación atmosférica en Ciudad Juárez, Chihuahua. Salud Publica Mex 2000;42:288-297.

24. Brunekreef B, D ockery DW, Krzyzanowski M. Epidemiologic studies on short-term effects of low levels of major ambient air pollution components. Environ Health Perspect 1995;103:S3-S13.

25. Gold DR, Damokosh AI, Pope III A, D ockery DW, McD onnell W F, Serrano $P$ et al. Particulate and ozone pollutant effect on respiratory function of children in Southwest Mexico City, 1999. Epidemiology 1999;10 (1):8-16.

26. Peters A, Goldstein IF, Beyer U, Franke K, Heinrich J, D ockery W. Acute health effects of exposure to high levels of air pollution in Eastern Europe. Am J Epidemiol 1996;144(6):570-581.

27. Roemer W, Hoek $G$, Brunekreef B. Effect of ambient winter air pollution on respiratory health of children with chronic respiratory symptoms. Am Rev Respir Dis 1993;147:118-124.

28. Schwartz J.Air pollution and hospital admissions for heart disease in eight US counties. Epidemiology 1999;10(1):17-22.

29. Romieu I, Meneses F, Sienra-Monge JJ, Huerta J, Ruiz S,W hite M et al. Effects of urban air pollutants on emergency visits for childhood asthma in Mexico City. Am J Epidemiol 1995;141:546-553. 
30. Peters A, D ockery DW, H einrich J,W ichmann HE. Short-term effects of particulate air pollution on respiratory morbidity in asthmatic children. Eur Respir J 1997;10:872-879.

31.Vedal S, Petkau J,W hite R, Blair J.Acute effects of ambient inhalable particles in asthmatic and nonasthmatic children living in Mexico City. Am J Respir Crit Care Med 1996;154:300-307.

32. D ab W, Medina S, Q uenel P, Le Moullec Y, Le Tertre A, Thelot B et al. Short term respiratory health effects of ambient air pollution: Results of the APHEA project in Paris. J Epidemiol Community Health 1996;50(Suppl 1):S542-S546.
33. Ponce de León A,Anderson HR, Bland JM, Strachan DP, Bower J. Effects of air pollution on daily hospital admissions for respiratory disease in London between 1987-88 and 1991-92.J Epidemiol Community Health 1996;50(Suppl 1):S63-S70.

34. Romieu I, Samet J, Smith KR, Bruce N. O utdoor air pollution and acute respiratory infections among children in developing countries. J 0 ccup Environ Med 2002;44:640-649. 